\title{
A palette of approaches for adiabatic elimination in bipartite open quantum systems with Hamiltonian dynamics on target
}

\author{
Paolo Forni ${ }^{1}$, Timothée Launay ${ }^{1}$, Alain Sarlette ${ }^{2}$, and Pierre Rouchon ${ }^{1}$
}

\begin{abstract}
Adiabatic elimination is a model reduction technique commonly used by physicists to eliminate quickly dissipating components from quantum physics equations. We revisit this technique when the target non-dissipating component is driven by Hamiltonian actuation at a fast timescale. Following center manifold theory, we can still write reduced dynamics for the target component, but there may be new conditions to ensure that it takes the standard structure of quantum dynamics, i.e. evolution equations of Lindblad type and coordinate changes in Kraus map form. We here propose various approaches to recover a Lindblad form up to third-order terms: without conditions for finite-dimensional systems, and under some finite set conditions for infinite-dimensional systems.
\end{abstract}

\section{INTRODUCTION}

The Lindblad master equation is the quantum equivalent of dissipative systems, where in principle Hamiltonian interactions with the large outside world are summarized by dissipation operators (Born-Markov approximation, [4]). When engineering quantum systems, dissipative components are interconnected with Hamiltonian ones and the behavior of interest is the one after the fast dissipative transient has stabilized. We study the basic building block consisting of a target system weakly coupled with a strongly dissipative ancillary system. By exploiting a time-scale separation between the fast uncoupled dynamics and the weak interaction dynamics, the full system can be decoupled with high accuracy into a fast decaying component, approximately corresponding to the ancillary system, and a remaining component of lower dimension corresponding closely to the quantum system of interest. Physicists have routinely considered the dominant term of this approximation as "adiabatic elimination" of the fast dynamics.

This picture can be made more precise in a system theoretic context on the basis of geometric singular perturbation theory and center manifold theory, as introduced in [3], [2]. From a mathematical viewpoint an asymptotic series expansion at higher orders is clear since the Lindblad equation is linear - we are thus approximating the slowly decaying eigenvalues and eigenspaces of a linear system. However, to preserve an intrinsic physical meaning for the reduced system, the elimination should provide again a Lindblad form

\footnotetext{
${ }^{1}$ Centre Automatique et Systèmes, Mines-ParisTech, PSL Research University. 60 Bd Saint-Michel, 75006 Paris, France; and INRIA Paris, 2 rue Simone Iff, 75012 Paris, France. paolo.fornieinria.fr, timothee. launay@mines-paristech. fr, pierre.rouchon@mines-paristech.fr.

${ }^{2}$ INRIA Paris, 2 rue Simone Iff, 75012 Paris, France; and Ghent University / Electronics and Information Systems, Technologiepark 914, 9052 Zwijnaarde, Belgium. alain.sarlettedinria.fr.

This work has been supported by the ANR-JCJC project HAMROQS
}

in the reduced equations; and to easily view it as a component of the full world, the coordinate change mapping the reduced model into the complete one should be a completely positive trace preserving map, or Kraus map.

In [3], [2] general formulas are given that solve the series expansion, with Lindblad and Kraus form, up to second order included, when the center manifold $(\simeq$ target quantum system) corresponds to a set of steady states at zero order. However, quantum technology definitely aims to act on the target systems, possibly at fast time scales. This fast Hamiltonian action makes the center manifold correspond to the eigenspace of imaginary eigenvalues at zero order, and changes the computations towards recovering Lindblad form reduced dynamics. A first generalization in [8] takes into account fast Hamiltonian dynamics on target, under a precise condition linking the actuation and interaction Hamiltonians.

In the present paper we remove this condition: the actuation Hamiltonian, as well as the interaction Hamiltonian, are arbitrary. We study how the actuation influences the reduced model dynamics, and find an appropriate Kraus map.

The paper is organized as follows. Section II describes the abstract problem setting and Section III provides the adiabatic elimination formulas that can be used in presence of fast actuation. Section IV completes the presentation with two applications in ongoing quantum experiments. The technical proofs are given in appendix.

\section{SETTING}

\section{A. Bipartite system}

Open quantum systems are typically described by differential equations on the manifold of density operators $\rho \in \mathfrak{D}(\mathcal{H})$, namely the set of linear Hermitian nonnegative operators of trace one on a Hilbert space $\mathcal{H}$. We consider the composite Hilbert space $\mathcal{H}=\mathcal{H}_{A} \otimes \mathcal{H}_{B}$, where $\mathcal{H}_{A}$ is the auxiliary space and $\mathcal{H}_{B}$ is the target space. The evolution on $\mathcal{H}$ is then described by the Lindblad master equation [4]:

$$
\frac{d \rho}{d t}=\mathcal{L}_{A}(\rho)+\varepsilon \mathcal{L}_{\text {int }}(\rho)-i\left[\boldsymbol{H}_{B}, \rho\right],
$$

where $\epsilon$ is a small parameter emphasizing the time-scale separation; $\boldsymbol{H}_{B}$ is a Hermitian operator acting on $\mathcal{H}_{B}$ only; $\mathcal{L}_{A}$ is a Lindbladian super-operator acting on $\mathcal{H}_{A}$ only:

$$
\mathcal{L}_{A}(\rho):=-i\left[\boldsymbol{H}_{A}, \rho\right]+\sum_{\mu} \mathcal{D}\left[\boldsymbol{L}_{A, \mu}\right](\rho),
$$

with $\boldsymbol{L}_{A, \mu}$ a decoherence operator on $\mathcal{H}_{A}$ and $\mathcal{D}[.].($.$) the$ decoherence super-operator:

$$
\mathcal{D}[\boldsymbol{X}](\rho):=\boldsymbol{X} \rho \boldsymbol{X}^{\dagger}-\frac{1}{2}\left(\boldsymbol{X}^{\dagger} \boldsymbol{X}_{\rho}+\rho \boldsymbol{X}^{\dagger} \boldsymbol{X}\right) ;
$$


$\mathcal{L}_{\text {int }}$ is a Lindbladian super-operator that captures the interaction between $\mathcal{H}_{A}$ and $\mathcal{H}_{B}$, and here we assume that this interaction is Hamiltonian:

$$
\mathcal{L}_{\text {int }}(\rho)=-i\left[\sum_{k} \boldsymbol{A}_{k} \otimes \boldsymbol{B}_{k}^{\dagger}, \rho\right] .
$$

where $\boldsymbol{A}_{k}$ and $\boldsymbol{B}_{k}$ respectively are non-necessarilyHermitian operators acting on $\mathcal{H}_{A}$ and $\mathcal{H}_{B}$ only.

We assume that $\mathcal{L}_{A}$ is strongly dissipative and that there exists a unique density operator $\bar{\rho}_{A}$ such that $\mathcal{L}_{A}\left(\bar{\rho}_{A}\right)=0$. For $\varepsilon=0$, the system is uncoupled and the solutions stay separable into $\mathcal{H}_{A}$ and $\mathcal{H}_{B}$ at all times.

\section{B. Asymptotic expansion}

Adiabatic elimination of the strongly dissipative component $\mathcal{L}_{A}$ can be approached via center manifold theory by observing that, for $\varepsilon=0$, there exists an asymptotically stable center manifold $\mathcal{M}_{0}$ of same dimension as $\mathcal{H}_{B}$. Therefore, by virtue of Fenichel's Invariant Manifold Theorem [7], the existence of an asymptotically stable center manifold $\mathcal{M}_{\varepsilon}$ is persistent for all small enough $\varepsilon \neq 0$. An approximation of $\mathcal{M}_{\varepsilon}$ can be computed up to arbitrary precision (see Carr [5]). The fact that (1) is linear in $\rho$ further facilitates the obtention of guarantees and bounds.

The quantum particularity to adiabatic elimination is to preserve the structure of the reduced-order model, i.e. we aim to find:

- a Kraus ${ }^{1}$ map $\mathcal{K}_{\varepsilon}$ from $\rho_{s} \in \mathfrak{D}\left(\mathcal{H}_{s}\right)$ the reduced space with $\operatorname{dim}\left[\mathcal{H}_{s}\right]=\operatorname{dim}\left[\mathcal{H}_{B}\right]$ to the complete space $\rho \in \mathfrak{D}(\mathcal{H})$, such that $\mathcal{K}\left(\mathfrak{D}\left[\mathcal{H}_{s}\right]\right)$ yields $\mathcal{M}_{\varepsilon}$. Such map ensures that the reduced-order model $\rho_{s}$ still corresponds to density operators in the full physical space, thus $\rho_{s}$ retains physical meaning as directly describing measurement probabilities;

- the reduced dynamics on $\mathcal{H}_{s}$ in Lindblad form, i.e. $\frac{d}{d t} \rho_{s}=\mathcal{L}_{s, \varepsilon}\left(\rho_{s}\right)$ for some Lindbladian superoperator $\mathcal{L}_{s, \varepsilon}$.

Invariance of $\mathcal{M}_{\varepsilon}$ is then captured by the equation:

$\left.\mathcal{L}_{A}\left(\mathcal{K}_{\varepsilon}\left(\rho_{s}\right)\right)+\varepsilon \mathcal{L}_{i n t}\left(\mathcal{K}_{\varepsilon}\left(\rho_{s}\right)\right)-i\left[\boldsymbol{H}_{B}, \mathcal{K}_{\varepsilon}\left(\rho_{s}\right)\right)\right]=\mathcal{K}_{\varepsilon}\left(\mathcal{L}_{s, \varepsilon}\left(\rho_{s}\right)\right)$.

Carr's result [5] considers $\mathcal{K}_{\epsilon}, \mathcal{L}_{s, \epsilon}$ as infinite series in $\epsilon$ :

$$
\mathcal{K}_{\epsilon}\left(\rho_{s}\right)=\sum_{\ell=0}^{+\infty} \epsilon^{\ell} \mathcal{K}_{\ell}\left(\rho_{s}\right), \quad \mathcal{L}_{s, \epsilon}\left(\rho_{s}\right)=\sum_{\ell=0}^{+\infty} \epsilon^{\ell} \mathcal{L}_{s, \ell}\left(\rho_{s}\right) .
$$

By plugging (4) into (3) and identifying the terms of same order, an invariance relation is obtained at all orders. At zeroorder, we have:

$$
\mathcal{L}_{A}\left(\mathcal{K}_{0}\left(\rho_{s}\right)\right)-i\left[\boldsymbol{H}_{B}, \mathcal{K}_{0}\left(\rho_{s}\right)\right]=\mathcal{K}_{0}\left(\mathcal{L}_{s, 0}\left(\rho_{s}\right)\right)
$$

At first-order invariance, we get:

$$
\begin{aligned}
& \mathcal{L}_{A}\left(\mathcal{K}_{1}\left(\rho_{s}\right)\right)+\mathcal{L}_{\text {int }}\left(\mathcal{K}_{0}\left(\rho_{s}\right)\right)-i\left[\boldsymbol{H}_{B}, \mathcal{K}_{1}\left(\rho_{s}\right)\right] \\
& =\mathcal{K}_{0}\left(\mathcal{L}_{s, 1}\left(\rho_{s}\right)\right)+\mathcal{K}_{1}\left(\mathcal{L}_{s, 0}\left(\rho_{s}\right)\right)
\end{aligned}
$$

${ }^{1} \mathrm{~A}$ Kraus map takes the form $\mathcal{K}\left(\rho_{s}\right)=\sum_{l} \boldsymbol{M}_{l} \rho_{s} \boldsymbol{M}_{l}^{\dagger}$ for some operators $\boldsymbol{M}_{l}$, to ensure complete positivity [6], with $\sum_{l} \boldsymbol{M}_{l}^{\dagger} \boldsymbol{M}_{l}=$ identity for trace preservation. and at second-order:

$$
\begin{aligned}
& \mathcal{L}_{A}\left(\mathcal{K}_{2}\left(\rho_{s}\right)\right)+\mathcal{L}_{\text {int }}\left(\mathcal{K}_{1}\left(\rho_{s}\right)\right)-i\left[\boldsymbol{H}_{B}, \mathcal{K}_{2}\left(\rho_{s}\right)\right] \\
& =\mathcal{K}_{0}\left(\mathcal{L}_{s, 2}\left(\rho_{s}\right)\right)+\mathcal{K}_{1}\left(\mathcal{L}_{s, 1}\left(\rho_{s}\right)\right)+\mathcal{K}_{2}\left(\mathcal{L}_{s, 0}\left(\rho_{s}\right)\right) .
\end{aligned}
$$

\section{REDUCED MODEL FORMULAS}

In this section we provide solutions for the zero-, firstand second-order invariance equations, and consequently formulas for the reduced-order model of (1) up to thirdorder terms, in quantum form. The presence of $\boldsymbol{H}_{B}$ will be resolved in two possible ways. A first solution is proposed for systems where the spectrum of $\boldsymbol{H}_{B}$ is finite, thus in particular for any finite-dimensional systems. A second solution is proposed, possibly for infinite-dimensional systems, if some commutation relations end after a finite number of steps.

\section{A. Case of finite spectrum of $\boldsymbol{H}_{B}$}

In this section we make the following assumption.

Assumption 1: The spectrum of $\boldsymbol{H}_{B}$ consists of a finite set.

Assumption 1, together with hermiticity, implies the existence of an orthonormal basis of eigenvectors $\left\{\boldsymbol{x}_{i}\right\}_{i \in I}$ of $\boldsymbol{H}_{B}$ and its associated set of real eigenvalues $\left\{\lambda_{i}\right\}_{i \in I}$, for some index set $I$. It is then straightforward to show that the family of operators $\left\{\boldsymbol{x}_{i} \boldsymbol{x}_{j}^{\dagger}\right\}_{i, j \in I}$ represents an orthonormal basis for the Liouville space $\operatorname{End}\left[\mathcal{H}_{B}\right]$. Moreover, each $\boldsymbol{C}_{(i, j)}^{\dagger}:=\boldsymbol{x}_{i} \boldsymbol{x}_{j}^{\dagger}$ is an eigenoperator of $\left[\boldsymbol{H}_{B},.\right]$ with associated eigenvalue $c_{(i, j)}:=\lambda_{i}-\lambda_{j}$, in other words:

$$
\left[\boldsymbol{H}_{B}, \boldsymbol{C}_{(i, j)}^{\dagger}\right]=c_{(i, j)} \boldsymbol{C}_{(i, j)}^{\dagger}, \quad \boldsymbol{C}_{(i, j)} \boldsymbol{C}_{\left(i^{\prime}, j^{\prime}\right)}^{\dagger}=\delta_{i, i^{\prime}} \boldsymbol{C}_{\left(j, j^{\prime}\right)}^{\dagger} .
$$

where $\delta_{h, j}$ denotes the Kronecker delta. For brevity, we will equivalently index the sets over one index: $\boldsymbol{C}_{(i, j)}^{\dagger}=\boldsymbol{C}_{k}^{\dagger}$ and $c_{(i, j)}=c_{k}$.

Without loss of generality, we can still consider a Hamiltonian interaction in form (2) with the operators $\boldsymbol{B}_{k}$ now being (decomposed on the basis of) the eigenoperators of $\left[\boldsymbol{H}_{B}, \cdot\right]$ (, with possible weights factored into the $\boldsymbol{A}_{k}$ ). The zero-order (5) is immediately solved by setting:

$$
\mathcal{L}_{s, 0}\left(\rho_{s}\right)=-i\left[\boldsymbol{H}_{B}, \rho_{s}\right], \mathcal{K}_{0}\left(\rho_{s}\right)=\bar{\rho}_{A} \otimes \rho_{s} .
$$

At first order, let the Kraus map have the following structure:

$$
\begin{aligned}
\mathcal{K}\left(\rho_{s}\right) & =\mathcal{K}_{0}\left(\rho_{s}\right)+\epsilon \mathcal{K}_{1}\left(\rho_{s}\right)+\mathcal{O}\left(\epsilon^{2}\right) \\
& :=\left(\boldsymbol{I}-i \epsilon \sum_{k} \boldsymbol{M}_{k}\right)\left(\bar{\rho}_{A} \otimes \rho_{s}\right)\left(\boldsymbol{I}-i \epsilon \sum_{k} \boldsymbol{M}_{k}\right)^{\dagger}+\mathcal{O}\left(\epsilon^{2}\right),
\end{aligned}
$$

where the $M_{k}$ are operators to be defined in Theorem 1 .

Theorem 1: Consider model (1)-(2). Let Assumption 1 hold and, without loss of generality, $\boldsymbol{B}_{k}=\boldsymbol{C}_{k}$. Let $\boldsymbol{H}_{1}$ be an arbitrary Hermitian operator in $\operatorname{End}\left[\mathcal{H}_{B}\right]$, and $\left(f_{k}\right)$ its coordinates in the basis $\left\{\boldsymbol{C}_{k}^{\dagger}\right\}$. Then the first order invariance equation (6) is satisfied by the Lindbladian:

$$
\mathcal{L}_{s, 1}\left(\rho_{s}\right)=-i\left[\sum_{k} \operatorname{Tr}\left(\boldsymbol{A}_{k} \bar{\rho}_{a}\right) \boldsymbol{B}_{k}^{\dagger}-i\left[\boldsymbol{H}_{B}, \boldsymbol{H}_{1}\right], \rho_{s}\right]
$$

and by a map of the form (9) where $\boldsymbol{M}_{k}:=\boldsymbol{F}_{k} \otimes \boldsymbol{B}_{k}^{\dagger}$ and $\boldsymbol{F}_{k}$ is the unique solution of:

$$
\mathcal{L}_{A}\left(\boldsymbol{F}_{k} \bar{\rho}_{a}\right)+\mathcal{S}\left(-i c_{k} \boldsymbol{F}_{k} \bar{\rho}_{a}+\boldsymbol{A}_{k} \bar{\rho}_{a}\right)=0,
$$




$$
\operatorname{Tr}\left(\boldsymbol{F}_{k} \bar{\rho}_{a}\right)=f_{k},
$$

where $\mathcal{S}(\boldsymbol{X}):=\boldsymbol{X}-\operatorname{Tr}[\boldsymbol{X}] \bar{\rho}_{A}$.

Proof: See Appendix A.

To provide formulas for the second-order adiabatic elimination of model (1), we will build on a decomposition similar to [2]. Let $\boldsymbol{X}, \boldsymbol{Y}, \boldsymbol{Z}$ be the matrices whose entries are defined by (here ${ }^{\star}$ denotes complex conjugate):

$$
\begin{aligned}
x_{k^{\prime}, k} & :=z_{k^{\prime}, k}+z_{k, k^{\prime}}^{\star} \\
y_{k^{\prime}, k} & :=\frac{1}{2 i}\left(z_{k^{\prime}, k}-z_{k, k^{\prime}}^{\star}\right) \\
z_{k^{\prime}, k} & :=\operatorname{Tr}\left(\boldsymbol{P}_{k^{\prime}, k} \bar{\rho}_{a}\right)-\frac{i}{2}\left(c_{k}-c_{k^{\prime}}\right) \operatorname{Tr}\left(\boldsymbol{F}_{k} \bar{\rho}_{a} \boldsymbol{F}_{k^{\prime}}^{\dagger}\right) \\
\boldsymbol{P}_{k^{\prime}, k} & =\boldsymbol{A}_{k^{\prime}}^{\dagger} \boldsymbol{F}_{k}-\left(\operatorname{Tr}\left(\boldsymbol{A}_{k} \bar{\rho}_{a}\right)-i c_{k} f_{k}\right) \boldsymbol{F}_{\bar{k}^{\prime}} \\
\bar{k} & :=(j, i) \text { when } k=(i, j),
\end{aligned}
$$

and the $\boldsymbol{F}_{k}$ are solutions to (10). It is possible to show along the lines of [3, Lemma 7] that:

$$
x_{k^{\prime}, k}=\operatorname{Tr}\left[\sum_{\mu}\left[\boldsymbol{L}_{A, \mu}, \boldsymbol{F}_{k}\right] \bar{\rho}_{A}\left[\boldsymbol{L}_{A, \mu}, \boldsymbol{F}_{k^{\prime}}\right]^{\dagger}\right],
$$

and thus $\boldsymbol{X} \succeq 0$ which implies that there exists $\boldsymbol{\Lambda} \in \mathbb{C}^{K \times K}$ such that $\boldsymbol{X}=\boldsymbol{\Lambda} \boldsymbol{\Lambda}^{\dagger}$, i.e. $x_{k^{\prime}, k}=\sum_{p} \boldsymbol{\Lambda}_{k^{\prime}, p}\left(\boldsymbol{\Lambda}_{k, p}\right)^{\star}$.

Theorem 2: Consider model (1)-(2). Let Assumption 1 hold and, without loss of generality, $\boldsymbol{B}_{k}=\boldsymbol{C}_{k}$. Select $\mathcal{L}_{s, 1}$ and $\mathcal{K}_{1}$ according to Theorem 1 . Let $\boldsymbol{H}_{2}$ be an arbitrary Hermitian operator in $\operatorname{End}\left[\mathcal{H}_{B}\right]$. Then the second order invariance equation is satisfied by the Lindbladian:

$$
\mathcal{L}_{s, 2}\left(\rho_{s}\right)=-i\left[\boldsymbol{H}_{s, 2}-i\left[\boldsymbol{H}_{B}, \boldsymbol{H}_{2}\right], \rho_{s}\right]+\sum_{p} \mathcal{D}\left[\boldsymbol{L}_{p}\right]\left(\rho_{s}\right),
$$

where $\boldsymbol{H}_{s, 2}:=\sum_{k^{\prime}, k} y_{k^{\prime}, k} \boldsymbol{B}_{k^{\prime}} \boldsymbol{B}_{k}^{\dagger}$ and $\boldsymbol{L}_{p}:=\sum_{k} \boldsymbol{\Lambda}_{k, p}^{\star} \boldsymbol{B}_{k}^{\dagger}$. Furthermore, there exists an associated map $\mathcal{K}_{2}$ such that $\mathcal{K}\left(\rho_{s}\right)=\mathcal{K}_{0}\left(\rho_{s}\right)+\epsilon \mathcal{K}_{1}\left(\rho_{s}\right)+\epsilon^{2} \mathcal{K}_{2}\left(\rho_{s}\right)$ is CPTP up to thirdorder terms.

Proof: See Appendix B.

Remark 1: When solving the invariance equations, the choice of coordinate mapping between $\mathcal{H}_{s}$ and $\mathcal{H}$ is not unique. This offers various possibilities to be leveraged in jointly determining the final forms of $\mathcal{K}_{\varepsilon}$ and of the reduced dynamics $\mathcal{L}_{s, \varepsilon}$, and similarly in the series expansion. The Hamiltonians $\boldsymbol{H}_{1}$ and $\boldsymbol{H}_{2}$ in the above theorems explicitly express some possibilities offered by this gauge degree of freedom. We will by default take them zero in the below applications.

\section{B. Case of vanishing commutators}

Assumption 1 is often not feasible with infinitedimensional systems, like the quantum harmonic oscillator which is nevertheless a canonical system of interest. In these cases, one typically resorts to operator properties, typically commutation relations, and we propose to do the same for adiabatic elimination. In [8] we have exploited the particular property $\forall k \exists c_{k}:\left[\boldsymbol{H}_{B}, \boldsymbol{B}_{k}^{\dagger}\right]=c_{k} \boldsymbol{B}_{k}^{\dagger}$. In this section, we propose a complementary approach motivated by the example in Section IV-B. Let $\mathcal{C}^{h}(\cdot, \cdot)$ denote the repeated commutator:

$$
\mathcal{C}^{h}\left(\boldsymbol{X}_{1}, \boldsymbol{X}_{2}\right):=\underbrace{\left[\boldsymbol{X}_{1},\left[\boldsymbol{X}_{1},\left[\ldots, \boldsymbol{X}_{2}\right]\right]\right]}_{h \text { times }} .
$$

Assumption 2: There exists a finite $h \in \mathbb{N}$ such that $\mathcal{C}^{h}\left(\boldsymbol{H}_{B}, \boldsymbol{B}_{k}\right)=0$ for all $k \in\{1, \ldots K\}$.

For clarity of presentation, we will focus on the case $h=$ 3. Assumption 2 induces the following definitions: $\boldsymbol{C}_{k}:=$ $\left[\boldsymbol{H}_{B}, \boldsymbol{B}_{k}\right]$ and $\boldsymbol{D}_{k}:=\left[\boldsymbol{H}_{B}, \boldsymbol{C}_{k}\right]$, and for brevity ${ }^{(h)} \boldsymbol{B}_{k}:=$ $\mathcal{C}^{h}\left(\boldsymbol{H}_{B}, \boldsymbol{B}_{k}\right)$, e.g. $\boldsymbol{D}_{k}={ }^{(2)} \boldsymbol{B}_{k}$.

Theorem 3: Consider model (1)-(2). Let Assumption 2 hold with $h=3$. Then, the first-order invariance equation (6) is satisfied by the Lindbladian

$$
\mathcal{L}_{s, 1}\left(\rho_{s}\right)=-i\left[\boldsymbol{H}_{s, 1}, \rho_{s}\right],
$$

with $\boldsymbol{H}_{s, 1}:=\sum_{k} \operatorname{Tr}\left(\boldsymbol{A}_{k} \bar{\rho}_{a}\right) \boldsymbol{B}_{k}^{\dagger}$, and by a map $\mathcal{K}_{1}$ of the form (9) where $\boldsymbol{M}_{k}:=\sum_{h} \boldsymbol{F}_{k}^{h} \otimes{ }^{(h)} \boldsymbol{B}_{k}^{\dagger}$ and $\boldsymbol{F}_{k}^{h}$ is the unique solution of:

$$
\begin{aligned}
\mathcal{L}_{A}\left(\boldsymbol{F}_{k}^{\ell} \bar{\rho}_{A}\right)+\delta_{\ell, 0} \mathcal{S}\left(\boldsymbol{A}_{k} \bar{\rho}_{A}\right)+i\left(1-\delta_{\ell, 0}\right) \boldsymbol{F}_{k}^{\ell-1} \bar{\rho}_{A} & =0, \\
\operatorname{Tr}\left[\boldsymbol{F}_{k}^{\ell} \bar{\rho}_{A}\right] & =0
\end{aligned}
$$

for $\ell=0,1, \ldots, h$. Furthermore, $\mathcal{K}\left(\rho_{s}\right)$ is a CPTP map up to second-order terms.

Proof: See Appendix C.

Consider now the matrix $X \in \mathbb{C}^{K h \times K h}$ defined as:

$$
X_{k \ell, k^{\prime} \ell^{\prime}}:=\operatorname{Tr}\left[\left[\boldsymbol{L}_{A, \mu}, \boldsymbol{F}_{k}^{\ell}\right] \bar{\rho}_{A}\left[\boldsymbol{L}_{A, \mu}, \boldsymbol{F}_{k^{\prime}}^{\ell^{\prime}}\right]^{\dagger}\right]=: x_{k k^{\prime}}^{\ell \ell^{\prime}},
$$

for all $k=1, \ldots, K$ and $l=1, \ldots, h$. It is possible to show along the lines of [3, Lemma 7] that $X \succeq 0$ and thus there exists $\Lambda \in \mathbb{C}^{K h \times K h}$ such that $X=\Lambda \Lambda^{\dagger}$, i.e. $X k \ell, k^{\prime} \ell^{\prime}=$ $\sum_{p} \boldsymbol{\Lambda}_{k \ell, p}\left(\boldsymbol{\Lambda}_{k^{\prime} \ell^{\prime}, p}\right)^{\star}$.

Remark 2: By virtue of [3, Lemma 6] and equation (14), matrix elements $x_{k k^{\prime}}^{\ell \ell^{\prime}}$ may be also computed as follows:

$$
\begin{aligned}
& x_{k k^{\prime}}^{00}=\operatorname{Tr}\left[\boldsymbol{F}_{k}^{0} \bar{\rho}_{A} \boldsymbol{A}_{k^{\prime}}^{\dagger}+\boldsymbol{A}_{k} \bar{\rho}_{A} \boldsymbol{F}_{k^{\prime}}^{0 \dagger}\right] \\
& x_{k k^{\prime}}^{11}=i \operatorname{Tr}\left[\boldsymbol{F}_{k}^{0} \bar{\rho}_{A} \boldsymbol{F}_{k^{\prime}}^{1 \dagger}-\boldsymbol{F}_{k}^{1} \bar{\rho}_{A} \boldsymbol{F}_{k^{\prime}}^{0 \dagger}\right] \\
& x_{k k^{\prime}}^{22}=i \operatorname{Tr}\left[\boldsymbol{F}_{k}^{1} \bar{\rho}_{A} \boldsymbol{F}_{k^{\prime}}^{2 \dagger}-\boldsymbol{F}_{k}^{2} \bar{\rho}_{A} \boldsymbol{F}_{k^{\prime}}^{1 \dagger}\right] \\
& x_{k k^{\prime}}^{01}=i \operatorname{Tr}\left[\boldsymbol{F}_{k}^{0} \bar{\rho}_{A} \boldsymbol{F}_{k^{\prime}}^{0 \dagger}\right]+\operatorname{Tr}\left[\boldsymbol{A}_{k} \bar{\rho}_{A} \boldsymbol{F}_{k^{\prime}}^{1 \dagger}\right] \\
& x_{k k^{\prime}}^{02}=i \operatorname{Tr}\left[\boldsymbol{F}_{k}^{0} \bar{\rho}_{A} \boldsymbol{F}_{k^{\prime}}^{1 \dagger}\right]+\operatorname{Tr}\left[\boldsymbol{A}_{k} \bar{\rho}_{A} \boldsymbol{F}_{k^{\prime}}^{2^{\dagger}}\right] \\
& x_{k k^{\prime}}^{12}=i \operatorname{Tr}\left[\boldsymbol{F}_{k}^{0} \bar{\rho}_{A} \boldsymbol{F}_{k^{\prime}}^{2 \dagger}-\boldsymbol{F}_{k}^{1} \bar{\rho}_{A} \boldsymbol{F}_{k^{\prime}}^{1 \dagger}\right] .
\end{aligned}
$$

Theorem 4: Consider model (1)-(2). Let Assumption 2 hold with $h=3$. Then, the second-order invariance equation (7) is satisfied by Lindbladian:

$$
\begin{aligned}
\mathcal{L}_{s}\left(\rho_{s}\right)= & -i\left[\boldsymbol{H}_{B}+\varepsilon \boldsymbol{H}_{s, 1}+\varepsilon^{2} \boldsymbol{H}_{s, 2}, \rho_{s}\right] \\
& +\varepsilon^{2} \sum_{p} \mathcal{D}\left[\boldsymbol{L}_{p}\right]\left(\rho_{s}\right)+\mathcal{O}\left(\varepsilon^{3}\right),
\end{aligned}
$$

with $\boldsymbol{L}_{p}:=\sum_{k} \boldsymbol{\Lambda}_{k 0, p}^{\star} \boldsymbol{B}_{k}^{\dagger}+\boldsymbol{\Lambda}_{k 1, p}^{\star} \boldsymbol{C}_{k}^{\dagger}+\boldsymbol{\Lambda}_{k 2, p}^{\star} \boldsymbol{D}_{k}^{\dagger}$ and

$$
\boldsymbol{H}_{s, 2}:=\frac{1}{2 i} \sum_{k, k^{\prime}, \ell}\left(\operatorname{Tr}\left[\boldsymbol{A}_{k^{\prime}}^{\dagger} \boldsymbol{F}_{k}^{\ell} \bar{\rho}_{A}\right] \boldsymbol{B}_{k^{\prime}}{ }^{(\ell)} \boldsymbol{B}_{k}^{\dagger}-\text { herm. conj. }\right),
$$

with $\boldsymbol{H}_{s, 1}$ and the $\boldsymbol{F}_{k}^{\ell}$ computed as in Theorem 3. There exists an associated map $\mathcal{K}_{2}$, obtained from formula (28), 
such that $\mathcal{K}\left(\rho_{s}\right)=\left(\mathcal{K}_{0}+\varepsilon \mathcal{K}_{1}+\varepsilon \mathcal{K}_{2}\right)\left(\rho_{s}\right)$ is a CP map up to third-order terms and TP up to second-order terms.

Proof: See Appendix D.

\section{Applications}

We now present two examples of physical relevance that can be treated with our new formulas.

\section{A. Case of finite spectrum of $\boldsymbol{H}_{B}$}

The following example comes from [9], where a drive is applied to a so-called $\mathrm{V}$ three-level system and its transitions are monitored indirectly by dispersive interaction with a strongly dissipating harmonic oscillator ("cavity" or "resonator").

Let $\mathcal{H}_{A}$ and $\mathcal{H}_{B}$ respectively be the Hilbert space of the resonator and three-level system, respectively considered as the auxiliary and target component. The three levels are subsequently denoted as $|G\rangle$ ground, $|D\rangle$ dark, $|B\rangle$ bright. The so-called dark state is decoupled from its environment, and a small drive $\Omega_{D G}$ allows the state to transition from $|G\rangle$ to $|D\rangle$ and back. At the same time, the possible presence on $|G\rangle$ is monitored by a strong drive $\Omega_{B G}$ on its transition to $|B\rangle$, which is dispersively coupled with strength $\chi_{B}$ to a strongly dissipative resonator. In this way the resonator, probed at a frequency detuned by $\chi_{B}$ from its resonance, responds resonantly when the system is on the "bright" state. In the appropriate rotating frame and after rotating wave approximation, this setting corresponds to

$$
\begin{aligned}
\boldsymbol{H}_{B} & :=\left(\begin{array}{ccc}
0 & \Omega_{D G} & \Omega_{B G} \\
\Omega_{D G} & 0 & 0 \\
\Omega_{B G} & 0 & 0
\end{array}\right), \\
\boldsymbol{H}_{\text {int }} & :=\boldsymbol{a}^{\dagger} \boldsymbol{a}(|G\rangle\langle G|+| D\rangle\langle D|)
\end{aligned}
$$

with the matrix components ordered as $|G\rangle,|D\rangle,|B\rangle$; the Lindblad dynamics writes

$$
\frac{d \rho}{d t}=-i\left[u\left(\boldsymbol{a}^{\dagger}+\boldsymbol{a}\right)+\boldsymbol{H}_{B}+\chi_{B} \boldsymbol{H}_{i n t}, \rho\right]+\kappa \mathcal{D}[\boldsymbol{a}](\rho)
$$

with $u$ the strength of the coherent drive on the resonator mode, $\kappa$ the relaxation rate of the resonator and $\boldsymbol{a}$ the photon loss operator on the resonator.

A well-resolved observation of being on $|B\rangle\langle B|$, corresponds to $\chi_{m}>\kappa$. In contrast, our formulas allow to check what happens when this is not satisfied, i.e. when $\chi_{m} \ll \kappa$ (in practice a factor of 2 or 5 is sufficient to apply our formulas).

The commutator of $(|G\rangle\langle G|+| D\rangle\langle D|)$ with $\boldsymbol{H}_{B}$ does not allow us to apply the result of [8], but since $\mathcal{H}_{B}$ has finite dimension we can apply the results of Section III-A. We have $\bar{\rho}_{A}=|\alpha\rangle\langle\alpha|$ a coherent state, satisfying $\boldsymbol{a}|\alpha\rangle=\alpha|\alpha\rangle$, with $\alpha=-2 i u / \kappa$. This already gives $\boldsymbol{H}_{s, 1}=\chi_{B} \frac{4 u^{2}}{\kappa^{2}}(|G\rangle\langle G|+$ $|D\rangle\langle D|) \simeq-\chi_{B} \frac{4 u^{2}}{\kappa^{2}}|B\rangle\langle B|$ at first order, to be added to $\boldsymbol{H}_{s, 0}=\boldsymbol{H}_{B}$.

To get the second order, the decomposition of $\boldsymbol{H}_{B}$ into eigenvectors can be easily done by hand. Furthermore, we can observe that (10) is solved by

$$
\boldsymbol{F}_{k}=\frac{\alpha \chi_{B}}{i c_{k}+\kappa / 2}(\boldsymbol{a}-\alpha)^{\dagger},
$$

with $f_{k}=0$. From there straightforward (yet tedious) computations lead to the solution. Alternatively, we can use the formula (12) to directly obtain $X=\Lambda \Lambda^{\dagger}$ with $\Lambda$ a vector. This shows that we have a single decoherence channel, which we can analyze numerically. When taking small drives, our results are similar to those of previous papers, e.g. with $\Omega_{D G}=2 \cdot 10^{-5} \kappa$ and $\Omega_{B G}=2 \cdot 10^{-4} \kappa$ we have a dissipation operator

$$
\boldsymbol{L}_{1}=\frac{2|\alpha|}{\kappa}\left(\begin{array}{ccc}
1 & 0 & 0 \\
0 & 1 & 0 \\
0 & 0 & 0
\end{array}\right)
$$

Physically, this resembles the decoherence implied by a QND measurement distinguishing $|B\rangle$ from the two other states. When taking large drives, e.g. $\Omega_{D G}=0.2 \kappa$ and $\Omega_{B G}=2 \kappa$, our formulas are necessary and they indicate a switch in the measurement behavior:

$$
\boldsymbol{L}_{1}=\frac{2|\alpha|}{\kappa}\left(\begin{array}{ccc}
0.512 & -0.011 i & 0.062 i \\
0.011 i & 0.986 & -0.043 \\
-0.062 i & -0.043 & 0.501
\end{array}\right)
$$

Now, in agreement with the intuitive measurement principle, we are rather distinguishing whether the state is in $|D\rangle$, or oscillating somewhere between the two other states. In both cases, the measurement principle works according to this model, even if the spectral lines are not well resolved.

\section{B. Case of vanishing commutators}

The continuous-variable approach for protecting quantum information encodes a logical qubit (two-level system) in a quantum resonator (infinite number of levels). In particular, taking advantage of this redundancy via a reservoir engineering scheme, so-called cat qubits are an experimentally promising candidate [10]. More precisely, the target resonator is coupled via a two-photon process to an environment resonator such that the overall Lindbladian stabilizes the subspace $\operatorname{span}(|\beta\rangle,|-\beta\rangle)$ where $|\beta\rangle$ denotes the coherent state of amplitude $\beta$, satisfying $\boldsymbol{a}|\beta\rangle=\beta|\beta\rangle$. Hamiltonians must then be added to apply gates on this encoded information. Our formulas allow to investigate what happens when we push the speed of these gates to be as fast as the protection process.

Let $\mathcal{H}_{a}$ and $\mathcal{H}_{b}$ respectively be the Hilbert spaces of two resonators that are respectively modeling a highly dissipating component - used to evacuate entropy in the stabilization process - and the target "memory" component. Denote with $\boldsymbol{a}$ and $\boldsymbol{b}$ the annihilation operators in the first and second resonator mode respectively. By setting up an appropriate coupling and a constant drive on $\mathcal{H}_{a}$ [10], the coupled system can be engineered to follow:

$\frac{d}{d t} \rho=\kappa \mathcal{D}[\boldsymbol{a}](\rho)-i g\left[\boldsymbol{a}\left(\boldsymbol{b}^{2}-\beta^{2}\right)^{\dagger}+\boldsymbol{a}^{\dagger}\left(\boldsymbol{b}^{2}-\beta^{2}\right), \rho\right]-i\left[v^{\star} \boldsymbol{b}+v \boldsymbol{b}^{\dagger}, \rho\right]$

where $v$ is a drive meant to apply gates on the target system. The standard situation corresponds to $g, v \ll \kappa$. In this case all the small dynamics acts on $\mathcal{H}_{a}$ and we can apply the result in [3], yielding the reduced model:

$$
\frac{d}{d t} \rho_{s}=\frac{4 g^{2}}{\kappa} \mathcal{D}\left[\boldsymbol{b}^{2}-\beta^{2}\right]\left(\rho_{s}\right)-i\left[v^{\star} \boldsymbol{b}+v \boldsymbol{b}^{\dagger}, \rho_{s}\right] .
$$

When $v \ll 4 g^{2} / \kappa$, these dynamics effectively operate a gate [1] that rotates the interference fringes on the subspace 

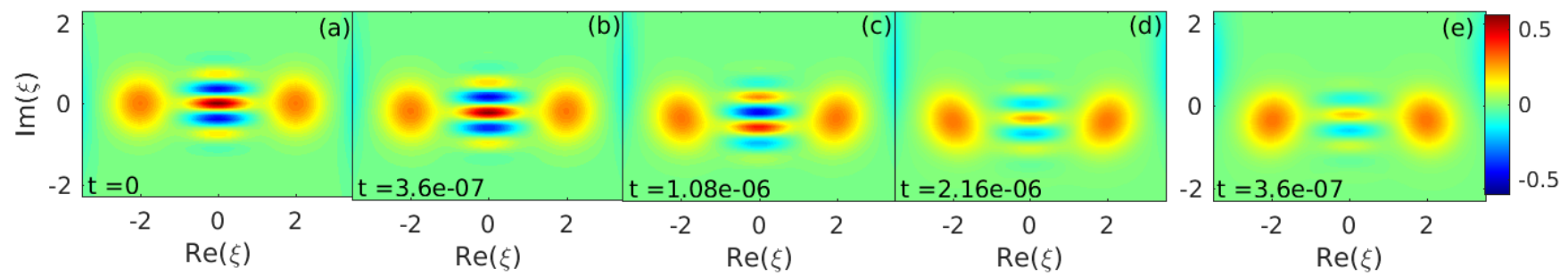

Fig. 1. Section IV-B: (a-d) Wigner functions of the state of resonator model (17) captured at four different times $t=0 \mu \mathrm{s}, 0.36 \mu \mathrm{s}, 1.08 \mu \mathrm{s} 2.16 \mu \mathrm{s}$, showing convergence to a fixed point; (e) Wigner function of the state of model (18) captured at $t=0.36 \mu \mathrm{s}$, showing that interference fringes have been lost. In both simulations, the initial density operator is: $\rho_{s}(0)=\left|\mathcal{C}_{\beta}^{+}\right\rangle\left\langle\mathcal{C}_{\beta}^{+}\right|$with $\left|\mathcal{C}_{\beta}^{+}\right\rangle:=(|\beta\rangle+|-\beta\rangle) / \mathcal{N}_{+}$where $\mathcal{N}_{+}$is a normalization constant and $\beta=2$; the parameter values are: $\kappa=1 \mathrm{MHz}, g=200 \mathrm{kHz}, v=500 \mathrm{kHz}$.

$\operatorname{span}(|\beta\rangle,|-\beta\rangle)$, at a rate proportional to $v$. The goal is to investigate how new effects must be taken into account when $v$ becomes of the same order as $\kappa$, thus limiting the achievable gate speed.

The model satisfies the hypothesis of Theorem 4 since system (16)-(2) is in the form (1) with, taking units such that $\kappa$ is of order 1: $\mathcal{L}_{A}:=\kappa \mathcal{D}[\boldsymbol{a}](\rho), \varepsilon:=g \ll 1, \boldsymbol{H}_{B}:=$ $v^{\star} \boldsymbol{b}+v \boldsymbol{b}^{\dagger}, \bar{\rho}_{A}:=|0\rangle\langle 0|, \boldsymbol{A}_{1}:=\boldsymbol{a}, \boldsymbol{A}_{2}:=\boldsymbol{a}^{\dagger}, \boldsymbol{B}_{1}:=\boldsymbol{b}^{2}-\beta^{2}$, $\boldsymbol{B}_{2}:=\left(\boldsymbol{b}^{2}-\beta^{2}\right)^{\dagger}, \boldsymbol{C}_{1}:=-2 v \boldsymbol{b}, \boldsymbol{C}_{2}:=2 v^{\star} \boldsymbol{b}^{\dagger}, \boldsymbol{D}_{1}:=2 v^{2}$, $\boldsymbol{D}_{2}:=2 v^{\star 2}$, and thus $\left[\boldsymbol{H}_{B}, \boldsymbol{D}_{k}\right]=0$. The associated $\boldsymbol{F}$ operators are computed via (14) as follows:

$$
\begin{aligned}
& \boldsymbol{F}_{1}^{0}=\boldsymbol{F}_{1}^{1}=\boldsymbol{F}_{1}^{2}=0, \boldsymbol{F}_{2}^{0}=\frac{2}{\kappa}|1\rangle\langle 0|, \\
& \boldsymbol{F}_{2}^{1}=i \frac{4}{\kappa^{2}}|1\rangle\left\langle 0\left|, \boldsymbol{F}_{2}^{2}=-\frac{8}{\kappa^{3}}\right| 1\right\rangle\langle 0| .
\end{aligned}
$$

Matrix $\boldsymbol{X}$ is computed via Remark 2 as follows:

$$
\boldsymbol{X}=\left(\begin{array}{ccc}
4 / \kappa & 0 & 0 \\
0 & 16 / \kappa^{3} & -32 i / \kappa^{4} \\
0 & 32 i / \kappa^{4} & 64 / \kappa^{5}
\end{array}\right)
$$

By Theorem 4 and after regrouping terms, the reduced model is:

$$
\begin{aligned}
\mathcal{L}_{s}\left(\rho_{s}\right)= & -i\left(1+\frac{32 g^{2}|v|^{2}}{\kappa^{4}}\right)\left[v^{\star} \boldsymbol{b}+v \boldsymbol{b}^{\dagger}, \rho_{s}\right] \\
& -i \frac{4 g^{2}}{\kappa^{2}}\left[H_{s, 2, c}, \rho_{s}\right] \\
& +\frac{4 g^{2}}{\kappa} \mathcal{D}\left[\boldsymbol{b}^{2}-\beta^{2}\right]\left(\rho_{s}\right)+\frac{32 g^{2}|v|^{2}}{\kappa^{3}} \mathcal{D}[\boldsymbol{b}]\left(\rho_{s}\right)+\mathcal{O}\left(g^{3}\right),
\end{aligned}
$$

with $H_{s, 2, c}=\left(v\left(\boldsymbol{b}^{2}-\beta^{2}\right)^{\dagger} \boldsymbol{b}+v^{\star} \boldsymbol{b}^{\dagger}\left(\boldsymbol{b}^{2}-\beta^{2}\right)\right)+\frac{2 i}{\kappa}\left(v^{2} \boldsymbol{b}^{\dagger 2}-\right.$ $\left.v^{\star 2} \boldsymbol{b}^{2}\right)$. When $v \ll \kappa$, neglecting higher-order terms gives back (17). For $v$ of the same order as $\kappa$, we see not only a modification of the Hamiltonian, which is reversible and thus in principle could be countered, but also a significant new decoherence channel in $\boldsymbol{b}$. Since $\boldsymbol{b}| \pm \beta\rangle= \pm \beta| \pm \beta\rangle$, this contributes to further blurring the interference fringes, as illustrated on Figure 1: model (18) converges to a fixed point without fringes in about 0.36 microseconds, while model (17) erroneously predicts that we would still have significant fringes at this time, and only lose them much later. We thus conclude that pushing with $v$ as strongly as $\kappa$ destroys the interference fringes very quickly, resulting in information loss.

\section{CONCLusions}

We have provided formulas for adiabatic elimination of a fast dissipating component connected to a target component, when the target component is subject to maybe slow dissipation but fast Hamiltonian actuation. Formulas are obtained for two cases: when actuation has a finite spectrum, and when it satisfies some iterated commutation relation. We have illustrated those methods on two examples, showing that they capture significant effects which should be taken into account in experiment design.

\section{APPENDIX}

\section{A. Proof of Theorem 1}

From (9), the map $\mathcal{K}_{1}$ would take the form:

$$
\mathcal{K}_{1}\left(\bar{\rho}_{a} \otimes \rho_{s}\right)=\sum_{k}-i \boldsymbol{F}_{k} \bar{\rho}_{a} \otimes \boldsymbol{B}_{k}^{\dagger} \rho_{s}+\text { herm. conj. }
$$

Existence and uniqueness of solutions $\boldsymbol{F}_{k}$ is proved along the lines of [3, Lemma 4] by observing that $\mathcal{L}_{A}(\cdot)-i c_{k} I d(\cdot)$ is still a bijection on traceless operators. For a superoperator $\mathcal{Q}$ define $\mathcal{V}(\mathcal{Q})(\boldsymbol{X}):=-i\left(\left[\boldsymbol{H}_{B}, \mathcal{Q}(\boldsymbol{X})\right]-\mathcal{Q}\left(\left[\boldsymbol{H}_{B}, \boldsymbol{X}\right]\right)\right)$. Super-operator $\mathcal{V}$ satisfies the property:

$$
\mathcal{V}(\boldsymbol{X} \cdot)(\rho)=-i\left[\boldsymbol{H}_{B}, \boldsymbol{X}\right] \rho .
$$

By considering the zero-order solution (8) and $\mathcal{K}_{1}$ from (19), and by making use of formulas (10) and (20), the first-order invariance equation (6) simplifies to:

$$
\begin{aligned}
\bar{\rho}_{A} \otimes & \mathcal{L}_{s, 1}\left(\rho_{s}\right)=\bar{\rho}_{A} \otimes \operatorname{Tr}_{A}\left[\mathcal{V}\left(\mathcal{K}_{1}\right)\left(\rho_{s}\right)\right. \\
& \left.-i\left[\sum_{k} \boldsymbol{A}_{k} \otimes \boldsymbol{B}_{k}^{\dagger}, \mathcal{K}_{0}\left(\rho_{s}\right)\right]\right] .
\end{aligned}
$$

By taking the partial trace over $\mathcal{H}_{A}$ over both sides of (21), we obtain the $\mathcal{L}_{s, 1}$ in the statement of Theorem 1.

It is immediate to see from (9) that $\mathcal{K}_{0}\left(\rho_{s}\right)+\varepsilon \mathcal{K}_{1}\left(\rho_{s}\right)$ is a completely positive map, as long as one can neglect the terms of order $\varepsilon^{2}$. It is also straightforward to see from (19) and property $f_{k} \in \mathbb{R}$ that $\operatorname{Tr}\left[\mathcal{K}_{1}\left(\rho_{s}\right)\right]=0$ for any $\rho_{s}$, thus $\mathcal{K}_{0}\left(\rho_{s}\right)+\varepsilon \mathcal{K}_{1}\left(\rho_{s}\right)$ is TP up to second-order terms. 


\section{B. Proof of Theorem 2}

First, we define the map $\mathcal{K}_{2}\left(\rho_{s}\right)$ and show that satisfies the second-order invariance equation (7). Let:

$$
\begin{aligned}
\mathcal{F}_{k, k^{\prime}}= & \sum_{\mu}\left[\boldsymbol{L}_{A, \mu}, \boldsymbol{F}_{k}\right] \bar{\rho}_{a}\left[\boldsymbol{L}_{A, \mu}, \boldsymbol{F}_{k^{\prime}}\right]^{\dagger}, \\
\mathcal{K}_{2}^{Q}\left(\rho_{s}\right)= & \int_{0}^{\infty} e^{t \mathcal{L}_{A}(.)}\left(\sum_{k, k^{\prime}} \mathcal{S}\left(e^{-i\left(c_{k}-c_{k^{\prime}}\right) t} \mathcal{F}_{k, k^{\prime}}\right)\right. \\
& \left.\otimes \boldsymbol{B}_{k}^{\dagger} \rho_{s} \boldsymbol{B}_{k^{\prime}}\right) d t+\bar{\tau} \bar{\rho}_{A} \otimes \mathcal{G}\left(\rho_{s}\right), \\
\mathcal{G}\left(\rho_{s}\right)= & \sum_{k, k^{\prime}} \delta_{c_{k}, c_{k^{\prime}}} \operatorname{Tr}\left[\mathcal{F}_{k, k^{\prime}}\right] \boldsymbol{B}_{k}^{\dagger} \rho_{s} \boldsymbol{B}_{k^{\prime}},
\end{aligned}
$$

where $\mathcal{S}(\boldsymbol{X}):=\boldsymbol{X}-\operatorname{Tr}[\boldsymbol{X}] \bar{\rho}_{A}$ and $\delta$ is the Kronecker delta. Now let $d_{0}, d_{1} \ldots d_{P}$ be a numeration of the eigenvalues of $\left[\boldsymbol{H}_{B},.\right]$ without repetitions with in particular $d_{0}=0$ and for any $k$ let $p(k)$ the index such that $d_{p(k)}=c_{k}$. Let $t_{0}=1$ and define the integration variables $t_{1}, t_{2} \ldots t_{P}$. We can check that:

$$
\begin{gathered}
\mathcal{G}\left(\rho_{s}\right)= \\
\frac{d_{1}}{2 \pi} \int_{0}^{\frac{2 \pi}{d_{1}}} \ldots \frac{d_{P}}{2 \pi} \int_{0}^{\frac{2 \pi}{d_{P}}}\left(\sum_{k, k^{\prime}} \operatorname{Tr}\left(e^{-i\left(c_{k} t_{p(k)}-c_{k^{\prime}} t_{p\left(k^{\prime}\right)}\right)} \mathcal{F}_{k, k^{\prime}}\right)\right. \\
\left.\boldsymbol{B}_{k}^{\dagger} \rho_{s} \boldsymbol{B}_{k^{\prime}}\right) d t_{1} \ldots d t_{P} .
\end{gathered}
$$

Using this identity, it is possible to show along the lines of [8, Claim 2] that there exists $\bar{\tau}>0$ large enough such that $\mathcal{K}_{2}^{Q}(\cdot)$ is a completely positive map and the following property holds true :

$$
\mathcal{L}_{A}\left(\mathcal{K}_{2}^{Q}\left(\rho_{s}\right)\right)+\mathcal{S}_{A}\left(\mathcal{V}\left(\mathcal{K}_{2}^{Q}\right)\left(\rho_{s}\right)+\sum_{k, k^{\prime}} \mathcal{F}_{k, k^{\prime}} \otimes \boldsymbol{B}_{k}^{\dagger} \rho_{s} \boldsymbol{B}_{k^{\prime}}\right)=0,
$$

where $\mathcal{S}_{A}(\boldsymbol{X}):=\boldsymbol{X}-\bar{\rho}_{A} \otimes \operatorname{Tr}_{A}[\boldsymbol{X}]$. Now let $\boldsymbol{H}_{2}$ be an arbitrary Hermitian operator in $\mathcal{H}_{B}$ and $\left\{g_{k}\right\}_{k} \in \mathbb{R}$ its coordinates in the basis $\left\{\boldsymbol{B}_{k}^{\dagger}\right\}_{k}$, and define :

$$
u_{k^{\prime}, k}=u_{\left(i^{\prime}, j^{\prime}\right),(i, j)}:=\delta_{i^{\prime}, 0} \delta_{i, 0} g_{\left(j^{\prime}, j\right)} .
$$

Scalars $u_{k^{\prime}, k}$ satisfy the property: $\sum_{k^{\prime}, k} u_{k^{\prime}, k} \boldsymbol{B}_{k^{\prime}} \boldsymbol{B}_{k}^{\dagger}=\boldsymbol{H}_{2}$. Now define $\mathcal{K}_{2}^{L}\left(\rho_{s}\right)=-\boldsymbol{N}\left(\bar{\rho}_{A} \otimes \rho_{s}\right):=\sum_{k, k^{\prime}} \boldsymbol{U}_{k^{\prime}, k} \bar{\rho}_{A} \otimes$ $\boldsymbol{B}_{k^{\prime}} \boldsymbol{B}_{k}^{\dagger} \rho_{s}$ where $\boldsymbol{U}_{k^{\prime}, k}$ is the unique operator satisfying:

$\mathcal{L}_{A}\left(\boldsymbol{U}_{k^{\prime}, k} \bar{\rho}_{A}\right)+\mathcal{S}\left(-i\left(c_{k}-c_{k^{\prime}}\right) \boldsymbol{U}_{k^{\prime}, k} \bar{\rho}_{A}-\boldsymbol{P}_{k^{\prime}, k} \bar{\rho}_{A}\right)=0$

$\operatorname{Tr}\left(\boldsymbol{U}_{k^{\prime}, k} \bar{\rho}_{A}\right)=-\frac{1}{2} \operatorname{Tr}\left(\boldsymbol{F}_{k} \bar{\rho}_{A} \boldsymbol{F}_{k^{\prime}}^{\dagger}\right)+i u_{k^{\prime}, k}-\delta_{c_{k}, c_{k^{\prime}}} \frac{\bar{\tau}}{2} \operatorname{Tr}\left[\mathcal{F}_{k, k^{\prime}}\right]$

Consider the CP map:

$$
\begin{aligned}
\mathcal{K}\left(\rho_{s}\right)= & \mathcal{K}_{0}\left(\rho_{s}\right)+\epsilon \mathcal{K}_{1}\left(\rho_{s}\right)+\epsilon^{2} \mathcal{K}_{2}\left(\rho_{s}\right)+\mathcal{O}\left(\epsilon^{3}\right) \\
:= & \left(\boldsymbol{I}-i \epsilon \sum_{k} \boldsymbol{M}_{k}-\varepsilon^{2} \boldsymbol{N}\right)\left(\bar{\rho}_{A} \otimes \rho_{s}\right)(\text { herm. conj. }) \\
& +\epsilon^{2} \mathcal{K}_{2}^{Q}\left(\rho_{s}\right)+\mathcal{O}\left(\epsilon^{3}\right) .
\end{aligned}
$$

We can verify that:

$$
\begin{aligned}
\operatorname{Tr}\left(\mathcal{K}_{2}\right)= & \operatorname{Tr}\left(\sum_{k, k^{\prime}} \boldsymbol{U}_{k^{\prime}, k} \bar{\rho}_{A} \otimes \boldsymbol{B}_{k^{\prime}} \boldsymbol{B}_{k}^{\dagger} \rho_{s}+\right.\text { herm. conj. } \\
& +\bar{\tau} \bar{\rho}_{A} \otimes \sum_{k, k^{\prime}} \delta c_{k}, c_{k^{\prime}} \operatorname{Tr}\left[\mathcal{F}_{k, k^{\prime}}\right] \boldsymbol{B}_{k}^{\dagger} \rho_{s} \boldsymbol{B}_{k^{\prime}} \\
& \left.+\sum_{k, k^{\prime}} \boldsymbol{F}_{k} \bar{\rho}_{A} \boldsymbol{F}_{k^{\prime}}^{\dagger} \otimes \boldsymbol{B}_{k}^{\dagger} \rho_{s} \boldsymbol{B}_{k^{\prime}}\right) \\
= & \sum_{k, k^{\prime}}\left(\operatorname{Tr}\left(\boldsymbol{U}_{k^{\prime}, k} \bar{\rho}_{A}\right)+\operatorname{Tr}\left(\boldsymbol{U}_{k, k^{\prime}} \bar{\rho}_{A}\right)^{\star}+\operatorname{Tr}\left(\boldsymbol{F}_{k} \bar{\rho}_{A} \boldsymbol{F}_{k^{\prime}}^{\dagger}\right)\right.
\end{aligned}
$$

$$
\left.=0, \quad+\bar{\tau} \delta_{c_{k}, c_{k^{\prime}}} \operatorname{Tr}\left[\mathcal{F}_{k, k^{\prime}}\right]\right) \operatorname{Tr}\left(\boldsymbol{B}_{k^{\prime}} \boldsymbol{B}_{k}^{\dagger} \rho_{s}\right)
$$

where we used the cycle property of the trace and then the definition of $\boldsymbol{U}_{k^{\prime}, k}$ and the hermiticity of $\boldsymbol{H}_{2}$. Hence we proved $\mathcal{K}$ is trace preserving up to third order terms. Moreover, it is possible to show that, by making use of maps $\mathcal{K}_{0}, \mathcal{K}_{1}, \mathcal{K}_{2}$ as in (25), maps $\mathcal{L}_{s, 0}, \mathcal{L}_{s, 1}$ as in (1), and [3, Lemma 6], the second-order invariance equation (7) is satisfied with:

$$
\begin{aligned}
\mathcal{L}_{s, 2}\left(\rho_{s}\right)=\sum_{k, k^{\prime}} & -\left(z_{k^{\prime}, k}+\left(c_{k}-c_{k^{\prime}}\right) u_{k^{\prime}, k}\right) \boldsymbol{B}_{k^{\prime}} \boldsymbol{B}_{k}^{\dagger} \rho_{s} \\
& +z_{k^{\prime}, k} \boldsymbol{B}_{k}^{\dagger} \rho_{s} \boldsymbol{B}_{k^{\prime}}+\text { herm. conj. }
\end{aligned}
$$

By making use of decomposition $x_{k^{\prime}, k}=\sum_{p} \boldsymbol{\Lambda}_{k^{\prime}, p} \boldsymbol{\Lambda}_{k, p}^{\star}$, the previous expression simplifies into:

$$
\begin{gathered}
\mathcal{L}_{s, 2}\left(\rho_{s}\right)=-i\left[\sum_{k^{\prime}, k}\left(y_{k^{\prime}, k}-i\left(c_{k}-c_{k^{\prime}}\right) u_{k^{\prime}, k}\right) \boldsymbol{B}_{k^{\prime}} \boldsymbol{B}_{k}^{\dagger}, \rho_{s}\right] \\
+\sum_{k^{\prime}, k, p} \eta_{k, p}^{\star} \eta_{k^{\prime}, p}\left(\boldsymbol{B}_{k}^{\dagger} \rho_{s} \boldsymbol{B}_{k^{\prime}}-\frac{1}{2}\left(\boldsymbol{B}_{k^{\prime}} \boldsymbol{B}_{k}^{\dagger} \rho_{s}+\rho_{s} \boldsymbol{B}_{k^{\prime}} \boldsymbol{B}_{k}^{\dagger}\right)\right),
\end{gathered}
$$

which readily gives our expression for $\mathcal{L}_{s, 2}$ in Theorem 2 .

\section{Proof of Theorem 3}

From the definitions of ${ }^{(h)} \boldsymbol{B}_{k}, \boldsymbol{C}_{k}$, and $\boldsymbol{D}_{k}$, we have: ${ }^{(h+1)} \boldsymbol{B}_{k}=\left[\boldsymbol{H}_{B},{ }^{(h)} \boldsymbol{B}_{k}\right]$ and ${ }^{(h+1)} \boldsymbol{B}_{k}^{\dagger}=-\left[\boldsymbol{H}_{B},{ }^{(h)} \boldsymbol{B}_{k}^{\dagger}\right]$. By plugging the zero-order solution (8) and map $\mathcal{K}_{1}\left(\rho_{s}\right)$ from (9) into the first order invariance equation (6), and by making use of (14), we obtain:

$$
\begin{aligned}
\sum_{k} & -i \operatorname{Tr}\left[\boldsymbol{A}_{k} \bar{\rho}_{A}\right] \otimes \boldsymbol{B}_{k}^{\dagger} \rho_{s} \\
& +\operatorname{Tr}\left[\boldsymbol{F}_{k}^{0} \bar{\rho}_{A}\right] \bar{\rho}_{A} \otimes \boldsymbol{C}_{k}^{\dagger} \rho_{s} \\
& +\operatorname{Tr}\left[\boldsymbol{F}_{k}^{1} \bar{\rho}_{A}\right] \bar{\rho}_{A} \otimes \boldsymbol{D}_{k}^{\dagger} \rho_{s} \\
& + \text { herm. conj. }=\bar{\rho}_{A} \otimes \mathcal{L}_{s, 1}\left(\rho_{s}\right) .
\end{aligned}
$$

By considering $\operatorname{Tr}\left[\boldsymbol{F}_{k}^{h} \bar{\rho}_{A}\right]=0$ and by taking the partial trace over $\mathcal{H}_{A}$ in (27), we immediately obtain $\mathcal{L}_{s, 1}\left(\rho_{s}\right)$ as in the Theorem 3 and thus (6) is satisfied. By taking the trace of $\mathcal{K}_{1}\left(\rho_{s}\right)$ from (9) we also get: $\operatorname{Tr}\left[\mathcal{K}_{1}\left(\rho_{s}\right)\right]$

$$
=-i \sum_{k, h} \operatorname{Tr}\left[\boldsymbol{F}_{k}^{h} \bar{\rho}_{A}\right] \operatorname{Tr}\left[{ }^{(h)} \boldsymbol{B}_{k}^{\dagger} \rho_{s}\right]+\text { herm. conj. }=0,
$$

which proves that $\mathcal{K}$ is TP up to second-order terms.

\section{Proof of Theorem 4}

First, we define the map $\mathcal{K}_{2}\left(\rho_{s}\right)$ and show that it satisfies the second-order invariance equation (7). Let:

$$
\begin{aligned}
& \mathcal{Q}_{t}\left(\bar{\rho}_{A}, \rho_{s}\right):= \sum_{\substack{k, k^{\prime} \in\{1, \ldots, K\} \\
h, h^{\prime} \in\{0, \ldots, M\}}}\left[\boldsymbol{L}_{A, \mu}, \boldsymbol{F}_{k}^{h}\right] \bar{\rho}_{A}\left[\boldsymbol{L}_{A, \mu}, \boldsymbol{F}_{k^{\prime}}^{h^{\prime}}\right]^{\dagger} \\
& \otimes{ }^{(h)} \boldsymbol{B}_{k}^{\dagger}\left(e^{+i\left[\boldsymbol{H}_{B}, \ldots\right] t}\left(\rho_{s}\right)\right){ }^{\left(h^{\prime}\right)} \boldsymbol{B}_{k^{\prime}} \\
& \mathcal{G}\left(\rho_{s}\right):= \int_{0}^{T} \operatorname{Tr}_{A}\left[\mathcal{Q}_{t}\left(\bar{\rho}_{A}, \rho_{s}\right)\right] d t \\
& \mathcal{K}_{2}^{Q}\left(\rho_{s}\right)= \int_{0}^{+\infty} e^{\mathcal{L}_{A}(\cdot) t-i\left[\boldsymbol{H}_{B}, \cdot\right] t}\left(\mathcal{S}\left(\mathcal{Q}_{t}\left(\bar{\rho}_{A}, \rho_{s}\right)\right)\right) d t \\
&+\bar{\tau} \bar{\rho}_{A} \otimes \mathcal{G}\left(\rho_{s}\right)
\end{aligned}
$$


It is possible to show along the lines of [8, Claim 2] that there exists $T>0$ and $\bar{\tau}>0$ large enough such that $\mathcal{K}_{2}^{Q}(\cdot)$ is a completely positive map and the following property holds true:

$$
\begin{aligned}
& \mathcal{L}_{A}\left(\mathcal{K}_{2}^{Q}\left(\rho_{s}\right)\right)-i\left[\boldsymbol{H}_{B}, \mathcal{K}_{2}^{Q}\left(\rho_{s}\right)\right]+i \mathcal{K}_{2}^{Q}\left(\left[\boldsymbol{H}_{B}, \rho_{s}\right]\right) \\
& +\mathcal{S}\left(\sum_{\mu} \sum_{\substack{k, k^{\prime} \in\{1, \ldots, K\} \\
h, h^{\prime} \in\{0,1,2\}}}\left[\boldsymbol{L}_{A, \mu}, \boldsymbol{F}_{k}^{h}\right] \bar{\rho}_{A}\left[\boldsymbol{L}_{A, \mu}, \boldsymbol{F}_{k^{\prime}}^{h^{\prime}}\right]^{\dagger}\right. \\
& \left.\quad \otimes{ }^{(h)} \boldsymbol{B}_{k}^{\dagger} \rho_{s}{ }^{\left(h^{\prime}\right)} \boldsymbol{B}_{k^{\prime}}\right)=\mathcal{O}(\varepsilon) .
\end{aligned}
$$

Now let $\boldsymbol{E}_{k}$ be the possibly non-unique operator such that: $\left[\boldsymbol{H}_{B}, \boldsymbol{E}_{k}\right]=\boldsymbol{B}_{k}$. Denote $f_{k k^{\prime}}^{h h^{\prime}}:=\operatorname{Tr}\left[\boldsymbol{F}_{k}^{h} \bar{\rho}_{A} \boldsymbol{F}_{k^{\prime}}^{h^{\prime} \dagger}\right]$ and $\mathcal{C}_{\boldsymbol{X}}^{h}:=\mathcal{C}^{h}\left(\boldsymbol{H}_{B}, \boldsymbol{X}\right)$. Define the operator:

$$
\begin{aligned}
& \mathcal{K}_{2}^{L}\left(\rho_{s}\right)=-\boldsymbol{N}\left(\bar{\rho}_{A} \otimes \rho_{s}\right)+\text { herm. conj. } \\
& \quad:=\sum_{k, k^{\prime}, w}-\boldsymbol{U}_{k k^{\prime}, w}^{h} \bar{\rho}_{A} \otimes \mathcal{C}_{\boldsymbol{B}_{k^{\prime}}{ }^{(h)} \boldsymbol{B}_{k}^{\dagger} \rho_{s}} \\
& \quad+\alpha_{k}^{\star} \boldsymbol{V}_{k^{\prime}, w}^{h} \bar{\rho}_{A} \otimes \mathcal{C}_{(h)}^{w} \boldsymbol{B}_{k^{\prime}}^{\dagger} \boldsymbol{B}_{k} \rho_{s}-w_{k k^{\prime}}^{1} \bar{\rho}_{A} \otimes \boldsymbol{E}_{k^{\prime}} \boldsymbol{D}_{k}^{\dagger} \rho_{s} \\
& \quad-w_{k k^{\prime}}^{2} \bar{\rho}_{A} \otimes \boldsymbol{D}_{k^{\prime}} \boldsymbol{E}_{k}^{\dagger} \rho_{s}-y_{k k^{\prime}}^{1} \bar{\rho}_{A} \otimes \boldsymbol{B}_{k^{\prime}} \boldsymbol{D}_{k}^{\dagger} \rho_{s} \\
& \quad-y_{k k^{\prime}}^{2} \bar{\rho}_{A} \otimes \boldsymbol{D}_{k^{\prime}} \boldsymbol{B}_{k}^{\dagger} \rho_{s}-z_{k k^{\prime}} \bar{\rho}_{A} \otimes \boldsymbol{C}_{k^{\prime}} \boldsymbol{D}_{k}^{\dagger} \rho_{s}+\text { herm. conj. },
\end{aligned}
$$

where $\boldsymbol{U}_{k, k^{\prime}, w}$ and $\boldsymbol{V}_{k^{\prime}, w}$ are the unique operators satisfying:

$$
\begin{aligned}
& \mathcal{L}_{A}\left(\boldsymbol{U}_{k, k^{\prime}, w}^{h} \bar{\rho}_{A}\right)+\delta_{w, 0} \mathcal{S}\left(\boldsymbol{A}_{k^{\prime}}^{\dagger} \boldsymbol{F}_{k}^{h} \bar{\rho}_{A}\right) \\
& \quad-i\left(1-\delta_{w, 0}\right) \mathcal{S}\left(\boldsymbol{U}_{k, k^{\prime}, w-1}^{h} \bar{\rho}_{A}\right)=0, \\
& \mathcal{L}_{A}\left(\boldsymbol{V}_{k^{\prime}, w}^{h} \bar{\rho}_{A}\right)+\delta_{w, 0} \boldsymbol{F}_{k^{\prime}}^{h} \bar{\rho}_{A} \\
& \quad-i\left(1-\delta_{w, 0}\right) \mathcal{S}\left(\boldsymbol{V}_{k^{\prime}, w}^{h} \bar{\rho}_{A}\right)=0, \\
& \operatorname{Tr}\left[\boldsymbol{U}_{k, k^{\prime}, 0}^{0} \bar{\rho}_{A}\right]=-\frac{1}{2} f_{k k^{\prime}}^{00}, \operatorname{Tr}\left[\boldsymbol{U}_{k, k^{\prime}, 1}^{0} \bar{\rho}_{A}\right]=\frac{1}{4}\left(f_{k k^{\prime}}^{01}-f_{k k^{\prime}}^{10}\right), \\
& \operatorname{Tr}\left[\boldsymbol{U}_{k, k^{\prime}, 2}^{0} \bar{\rho}_{A}\right]=\operatorname{Tr}\left[\boldsymbol{U}_{k, k^{\prime}, 3}^{0} \bar{\rho}_{A}\right]=0 \\
& \operatorname{Tr}\left[\boldsymbol{U}_{k, k^{\prime}, \star}^{1} \bar{\rho}_{A}\right]=\operatorname{Tr}\left[\boldsymbol{U}_{k, k^{\prime}, \star}^{2} \bar{\rho}_{A}\right]=0,
\end{aligned}
$$

and where:

$$
\begin{aligned}
w_{k k^{\prime}}^{1} & :=\frac{3}{4} f_{k k^{\prime}}^{10}-\frac{1}{4} f_{k k^{\prime}}^{01}, w_{k k^{\prime}}^{2}:=\frac{3}{4} f_{k k^{\prime}}^{01} \\
z_{k k^{\prime}} & :=-\frac{1}{2}\left(f_{k k^{\prime}}^{12}-f_{k k^{\prime}}^{21}\right), y_{k k^{\prime}}^{1}:=\frac{1}{2} i x_{k k^{\prime}}^{21}, y_{k k^{\prime}}^{2}:=-\frac{1}{2} i x_{k k^{\prime}}^{12}
\end{aligned}
$$

Consider the CP map:

$$
\begin{aligned}
\mathcal{K}\left(\rho_{s}\right)= & \mathcal{K}_{0}\left(\rho_{s}\right)+\epsilon \mathcal{K}_{1}\left(\rho_{s}\right)+\epsilon^{2} \mathcal{K}_{2}\left(\rho_{s}\right)+\mathcal{O}\left(\epsilon^{3}\right) \\
:= & \left(\boldsymbol{I}-i \epsilon \sum_{k} \boldsymbol{M}_{k}-\varepsilon^{2} \boldsymbol{N}\right)\left(\bar{\rho}_{A} \otimes \rho_{s}\right)(\text { herm. conj. }) \\
& +\mathcal{K}_{2}^{Q}\left(\rho_{s}\right)+\mathcal{O}\left(\epsilon^{3}\right) .
\end{aligned}
$$

By making use of $\mathcal{K}_{0}, \mathcal{K}_{1}, \mathcal{K}_{2}$ as in (28), $\mathcal{L}_{s, 0}, \mathcal{L}_{s, 1}$ as in (3), and [3, Lemma 6], the second-order invariance equation (7) reads as:

$$
\begin{aligned}
& \bar{\rho}_{A} \otimes \mathcal{L}_{s, 2}\left(\rho_{s}\right)= \\
& =-\sum_{\substack{k, k^{\prime} \\
h \in\{0,1,2\}}}\left(\operatorname{Tr}\left[\boldsymbol{A}_{k^{\prime}}^{\dagger} \boldsymbol{F}_{k}^{h} \bar{\rho}_{A}\right] \bar{\rho}_{A} \otimes \boldsymbol{B}_{k^{\prime}}{ }^{(h)} \boldsymbol{B}_{k}^{\dagger} \rho_{s}+\text { herm. conj. }\right) \\
& +\sum_{k, k^{\prime}, w=0}\left(+i \operatorname{Tr}\left[\boldsymbol{U}_{k k^{\prime}, w}^{h} \bar{\rho}_{A}\right] \bar{\rho}_{A} \otimes \mathcal{C}_{\boldsymbol{B}_{k^{\prime}}{ }^{(h+1} \boldsymbol{B}_{k}^{\dagger} \rho_{s}}^{1}\right. \\
& +i w_{k k^{\prime}}^{1} \bar{\rho}_{A} \otimes \boldsymbol{B}_{k^{\prime}} \boldsymbol{D}_{k}^{\dagger} \rho_{s}-i w_{k k^{\prime}}^{2} \bar{\rho}_{A} \otimes \boldsymbol{D}_{k^{\prime}} \boldsymbol{B}_{k}^{\dagger} \rho_{s} \\
& +i y_{k k^{\prime}}^{1} \bar{\rho}_{A} \otimes \boldsymbol{C}_{k^{\prime}} \boldsymbol{D}_{k}^{\dagger} \rho_{s}-i y_{k k^{\prime}}^{2} \bar{\rho}_{A} \otimes \boldsymbol{D}_{k^{\prime}} \boldsymbol{C}_{k}^{\dagger} \rho_{s} \\
& \left.+i z_{k k^{\prime}} \bar{\rho}_{A} \otimes \boldsymbol{D}_{k^{\prime}} \boldsymbol{D}_{k}^{\dagger} \rho_{s}+\text { herm. conj. }\right)
\end{aligned}
$$

$$
+\sum_{\substack{k, k^{\prime} \in\{1, \ldots, K\} \\ h, h^{\prime} \in\{0,1,2\}}} x_{k k^{\prime}}^{h h^{\prime}} \bar{\rho}_{A} \otimes{ }^{(h)} \boldsymbol{B}_{k}^{\dagger} \rho_{s}{ }^{\left(h^{\prime}\right)} \boldsymbol{B}_{k^{\prime}} .
$$

Taking the partial trace over $\mathcal{H}_{A}$ on both sides of expression (29) yields:

$$
\begin{aligned}
& \mathcal{L}_{s, 2}\left(\rho_{s}\right)=-i \boldsymbol{H}_{s, 2} \rho_{s} \\
& +\sum_{\substack{k, k^{\prime} \in\{1, \ldots, K\} \\
h, h^{\prime} \in\{0,1,2\}}} \frac{x_{k k^{\prime}}^{h h^{\prime}}}{2}\left({ }^{(h)} \boldsymbol{B}_{k}^{\dagger} \rho_{s}{ }^{\left(h^{\prime}\right)} \boldsymbol{B}_{k^{\prime}}-{ }^{\left(h^{\prime}\right)} \boldsymbol{B}_{k^{\prime}}{ }^{(h)} \boldsymbol{B}_{k}^{\dagger} \rho_{s}\right) \\
& + \text { herm. conj.. }
\end{aligned}
$$

which readily simplifies to the $\mathcal{L}_{s, 2}$ in Theorem 4 by using the decomposition $\boldsymbol{X}_{k h, k^{\prime} h^{\prime}}=\sum_{p} \boldsymbol{\Lambda}_{k h, p} \boldsymbol{\Lambda}_{k^{\prime} h^{\prime}, p}^{\star}$.

\section{REFERENCES}

[1] R. Azouit, A. Sarlette, and P. Rouchon. Convergence and adiabatic elimination for a driven dissipative quantum harmonic oscillator. In 2015 54th IEEE Conference on Decision and Control (CDC), pages 6447-6453, Dec 2015.

[2] R. Azouit, A. Sarlette, and P. Rouchon. Adiabatic elimination for open quantum systems with effective lindblad master equations. In 2016 IEEE 55th Conference on Decision and Control (CDC), pages 4559-4565, Dec 2016

[3] Rémi Azouit, Francesca Chittaro, Alain Sarlette, and Pierre Rouchon. Towards generic adiabatic elimination for bipartite open quantum systems. Quantum Science and Technology, 2(4):044011, 2017.

[4] H.P. Breuer and F. Petruccione. The Theory of Open Quantum Systems. Oxford University Press, 2002

[5] J. Carr. Applications of Centre Manifold Theory. Applied Mathematical Sciences. Springer New York, 2012.

[6] Man-Duen Choi. Completely positive linear maps on complex matrices. Linear Algebra and its Applications, 10(3):285 - 290, 1975.

[7] Neil Fenichel. Geometric singular perturbation theory for ordinary differential equations. Journal of Differential Equations, 31(1):53 98, 1979.

[8] P. Forni, A. Sarlette, T. Capelle, E. Flurin, S. Deléglise, and P. Rouchon. Adiabatic elimination for multi-partite open quantum systems with non-trivial zero-order dynamics. In 2018 IEEE Conference on Decision and Control (CDC), pages 6614-6619, Dec 2018.

[9] ZK Minev, SO Mundhada, Shyam Shankar, Philip Reinhold, Ricardo Gutiérrez-Jáuregui, RJ Schoelkopf, Mazyar Mirrahimi, HJ Carmichael, and $\mathrm{MH}$ Devoret. To catch and reverse a quantum jump mid-flight. Nature, page 1, 2019

[10] Mazyar Mirrahimi, Zaki Leghtas, Victor V Albert, Steven Touzard, Robert J Schoelkopf, Liang Jiang, and Michel H Devoret. Dynamically protected cat-qubits: a new paradigm for universal quantum computation. New Journal of Physics, 16(4):045014, apr 2014. 